\title{
Associated Higgs boson + jets production at the LHC and Catani-Ciafaloni-Fiorani-Marchesini gluon dynamics in a proton
}

\author{
A. V. Lipatov ${ }^{1,2, *}$ and M. A. Malyshev $\circledast^{1}$ \\ ${ }^{1}$ Skobeltsyn Institute of Nuclear Physics, Lomonosov Moscow State University, 119991 Moscow, Russia \\ ${ }^{2}$ Joint Institute for Nuclear Research, 141980 Dubna, Moscow region, Russia
}

(Received 5 December 2020; accepted 23 April 2021; published 18 May 2021)

\begin{abstract}
We consider the associated production of Higgs boson and hadronic jet(s) in $p p$ collisions at the LHC for the first time using the $k_{T}$-factorization approach. Our analysis is based on the off-shell gluon-gluon fusion subprocess, where nonzero transverse momenta of initial gluons are taken into account and covers $H \rightarrow \gamma \gamma, H \rightarrow Z Z^{*} \rightarrow 4 l$ (with $l=e, \mu$ ), and $H \rightarrow W^{+} W^{-} \rightarrow e^{ \pm} \mu^{\mp} \nu \bar{\nu}$ decay channels. The transverse momentum dependent (TMD) (or unintegrated) gluon densities in a proton are taken from Catani-Ciafaloni-Fiorani-Marchesini evolution equation. To simulate the kinematics of the produced jets, the TMD parton shower implemented into the Monte Carlo event generator CASCADE is applied. The comparison of our results with the latest experimental data taken by the CMS and ATLAS Collaborations at $\sqrt{s}=8$ and $13 \mathrm{TeV}$ is presented. Our predictions are compared also with the results of conventional higher-order perturbative QCD calculations. We highlight observables, which are sensitive to the TMD gluon densities in a proton.
\end{abstract}

DOI: 10.1103/PhysRevD.103.094021

Recently, the ATLAS and CMS Collaborations have presented measurements [1-8] of the total and differential cross sections of Higgs boson production in $p p$ collisions at the LHC conditions, both inclusive and associated with one or more hadronic jets. These data have been taken for $H \rightarrow \gamma \gamma, H \rightarrow Z Z^{*} \rightarrow 4 l$ (where $l=e$ or $\mu$ ), and $H \rightarrow$ $W^{+} W^{-} \rightarrow e^{ \pm} \mu^{\mp} \nu \bar{\nu}$ decay channels at the center-of-mass energies $\sqrt{s}=8$ and $13 \mathrm{TeV}$. Such measurements allow to probe fundamental properties of Higgs boson (e.g., spin and couplings to gauge bosons and fermions) and provide a testing ground for perturbative quantum chromodynamics (pQCD) predictions. Moreover, they can be used to investigate the gluon dynamics in a proton since the dominant mechanism of inclusive Higgs production is the gluon-gluon fusion (see, e.g., [9] and references therein).

The reported measurements [1-8] are found to be in good agreement with the next-to-next-to-leading-order (NNLO) pQCD predictions [10-15] obtained using HRES [16] and/or NNLOPS [17] Monte Carlo tools. Fixed-order perturbative QCD calculations are also available up to

\footnotetext{
*lipatov@theory.sinp.msu.ru
}

Published by the American Physical Society under the terms of the Creative Commons Attribution 4.0 International license. Further distribution of this work must maintain attribution to the author(s) and the published article's title, journal citation, and DOI. Funded by SCOAP.
NNLO [18-24]. ${ }^{1}$ Such NNLO calculations can be even improved at low transverse momenta by the soft-gluon resummation procedure, which has been carried out up to next-to-next-to-leading logarithmic accuracy [32,33]. An alternative description of the LHC data [1-8] can be achieved in the framework of the high-energy QCD factorization [34], or $k_{T}$-factorization [35]. This approach is based on the Balitsky-Fadin-Kuraev-Lipatov (BFKL) [36] or Catani-Ciafaloni-Fiorani-Marchesini (CCFM) [37] evolution equations, which resum large logarithmic terms proportional to $\alpha_{s}^{n} \ln ^{n} s \sim \alpha_{s}^{n} \ln ^{n} 1 / x$, important at high energies $\sqrt{s}$ (or, equivalently, at small longitudinal momentum fraction $x$ of the colliding proton carried by an interacting gluon). It can be understood since typical $x$ values are about of $x \sim m_{H} / \sqrt{s} \sim 0.008-0.015$ for Higgs mass $m_{H}=125 \mathrm{GeV}$ and $\sqrt{s}=13 \mathrm{TeV}$. Additionally, the CCFM equation takes into account terms proportional to $\alpha_{s}^{n} \ln ^{n} 1 /(1-x)$ and therefore can be applied for both small and large $x$ [37]. The $k_{T}$-factorization QCD approach has certain technical advantages in the ease of including higherorder $\mathrm{pQCD}$ radiative corrections (namely, main part of $\mathrm{NLO}+\mathrm{NNLO}+\ldots$ terms corresponding to real initialstate gluon emissions) in the form of transverse momentum dependent (TMD) (or unintegrated) gluon density and can

\footnotetext{
${ }^{1}$ The $\mathrm{N}^{3} \mathrm{LO}$ predictions for gluon-gluon fusion subprocess have become available recently $[25,26]$ and the NLO perturbative electroweak corrections to the Higgs production have been evaluated [27-31].
} 
be used as a convenient alternative to explicit higher-order pQCD calculations. The detailed description of this approach can be found, for example, in reviews [38,39].

The $k_{T}$-factorization formalism was already applied [40-49] to the inclusive Higgs boson production at the LHC. As it was demonstrated [46-49], this approach, being supplemented with the CCFM gluon dynamics, is able to describe the recent LHC data obtained at $\sqrt{s}=8$ and $13 \mathrm{TeV}$ in the diphoton, four-lepton, and $H \rightarrow W^{+} W^{-} \rightarrow e^{ \pm} \mu^{\mp} \nu \bar{\nu}$ decay channels even with leading-order off-shell (depending on the nonzero transverse momenta of incoming gluons) production amplitudes. Comparison with the higher-order pQCD calculations has been presented [49]. The sensitivity of the $k_{T}$-factorization predictions to the TMD gluon density specially is pointed out [49-51].

The associated production of Higgs boson and one or more hadronic jets is of special interest from different points of view. The most intriguing and remarkable point, in our opinion, is connected with the distinctive feature of the $k_{T^{-}}$ factorization approach regarding the final-state jet formation. While in the conventional (Dokshitzer-Gribov-LipatovAltarelli-Parisi (DGLAP)-based) parton-level pQCD, the produced jets are fully determined by corresponding hard scattering amplitude, in the $k_{T}$-factorization scenario in addition to the quarks and/or gluons produced in the hard subprocesses (which can form the hadronic jets), there are a number of gluons radiated in the course of their noncollinear evolution, which also give rise to final-state jets. So, the measured events with the detected jets could be useful in discrimination between the two calculation schemes. Therefore, it is of interest and importance to generate the $k_{T}$-factorization predictions for such events and test these predictions in as many cases as possible. Closely related to this is the selection of the TMD gluon densities in a proton best suited to describe the available experimental data. In the present note, we extend the previous consideration [41,46,49] of inclusive Higgs production to associated $H+$ jet(s) events. To correctly implement the kinematics of the final-state jets into our evaluations, the method of [52] is applied. This method is based on the reconstruction of CCFM evolution ladder using a TMD parton shower routine implemented into the Monte Carlo event generator CASCADE [53]. So, we essentially aim to make the comparison of our prediction with the available LHC data, thus hoping to extract some information about CCFM-evolved TMD gluon densities and to make some conclusions on their applicability for a description of such processes. Such calculations are performed for the first time.

Our main formulas were obtained in previous papers $[41,46,49]$. However, for the reader's convenience, let us very shortly describe the basic calculation steps. We start from the off-shell gluon fusion subprocesses

$$
g^{*}\left(k_{1}\right)+g^{*}\left(k_{2}\right) \rightarrow H(p) \rightarrow V\left(p_{1}\right)+V\left(p_{2}\right),
$$

where four-momenta of all particles are indicated in the parentheses and $V$ denotes $\gamma, W^{ \pm}$, or $Z$ bosons (any of the gauge bosons can decay into leptons and/or neutrino). It is important that both initial gluons carry nonzero transverse momenta: $\mathbf{k}_{1 T}^{2}=-k_{1 T}^{2} \neq 0, \mathbf{k}_{2 T}^{2}=-k_{2 T}^{2} \neq 0$. Using the effective Lagrangian for the Higgs coupling to gluons $[54,55]$ valid in the limit of infinite top quark mass, $m_{t} \rightarrow \infty$, one can easily obtain the corresponding off-shell production amplitudes. The latter can be written in a form [46,49] (see also [47])

$$
|\overline{\mathcal{A}}|^{2}=\frac{1}{1152 \pi^{4}} \alpha^{2} \alpha_{s}^{2} G_{F}^{2}|\mathcal{F}|^{2} \frac{\hat{s}^{2}\left(\hat{s}+\mathbf{p}_{T}^{2}\right)^{2}}{\left(\hat{s}-m_{H}^{2}\right)^{2}+m_{H}^{2} \Gamma_{H}^{2}} \cos ^{2} \phi
$$

for $H \rightarrow \gamma \gamma$ decay and

$$
\begin{aligned}
& |\overline{\mathcal{A}}|^{2}=\frac{512 \pi}{9} \alpha^{3} \alpha_{s}^{2} G_{F} \sqrt{2} m_{Z}^{2} C_{V} \frac{\left(\hat{s}+\mathbf{p}_{T}^{2}\right)^{2}}{\left(\hat{s}-m_{H}^{2}\right)^{2}+m_{H}^{2} \Gamma_{H}^{2}} \cos ^{2} \phi \\
& \times \frac{\left(g_{(V) L}^{4}+g_{(V) R}^{4}\right)\left(l_{1} \cdot l_{3}\right)\left(l_{2} \cdot l_{4}\right)+2 g_{(V) L}^{2} g_{(V) R}^{2}\left(l_{1} \cdot l_{4}\right)\left(l_{2} \cdot l_{3}\right)}{\left[\left(p_{1}^{2}-m_{V}^{2}\right)^{2}+m_{V}^{2} \Gamma_{V}^{2}\right]\left[\left(p_{2}^{2}-m_{V}^{2}\right)^{2}+m_{V}^{2} \Gamma_{V}^{2}\right]}
\end{aligned}
$$

for $H \rightarrow Z Z^{*} \rightarrow 4 l$ or $H \rightarrow W^{+} W^{-} \rightarrow e^{ \pm} \mu^{\mp} \nu \bar{\nu}$ decays. Here $G_{F}$ is the Fermi coupling constant, $l_{1}$ and $l_{3}$ are the gauge bosons decay leptons four-momenta, $l_{2}$ and $l_{4}$ are their antileptons four-momenta (so that $p_{1}=l_{1}+l_{2}$ and $\left.p_{2}=l_{3}+l_{4}\right), \quad \hat{s}=\left(k_{1}+k_{2}\right)^{2}, \quad \mathbf{p}_{T}=\mathbf{k}_{1 T}+\mathbf{k}_{2 T}$ is the transverse momentum of the Higgs boson, $\phi$ is the azimuthal angle between the transverse momenta of the initial off-shell gluons, $m_{V}$ and $\Gamma_{V}$ are the masses and decay widths of the corresponding particles. The exact expressions for $\mathcal{F}, C_{V}$, left and right weak current constants $g_{(V) L}$ and $g_{(V) R}$ are listed in [46,49] and all the calculation details are given there. The gauge invariant off-shell production amplitudes (2) and (3) have been implemented into the parton-level Monte Carlo event generator PEGASUS [56].

An important point of our calculations is connected with the proper determination of associated jets four-momenta. As it was noted above, the produced Higgs boson is accompanied by a number of gluons radiated in the course of the noncollinear evolution (in according to the CCFM equation), which give rise to final jets. To reconstruct the CCFM evolution ladder, we generate a Les Houches Event file [57] in the PEGASUS calculations and then process the file with a TMD shower tool implemented into the Monte Carlo event generator CASCADE [53]. In this way, one can consistently compute the cross section of associated $H+$ jet(s) production according to the experimental setup. ${ }^{2}$ The CCFM equation seems to be the most suitable

\footnotetext{
${ }^{2} \mathrm{~A}$ simplified model to implement the effects of parton showers into analytical calculations was used in earlier calculations [41].
} 

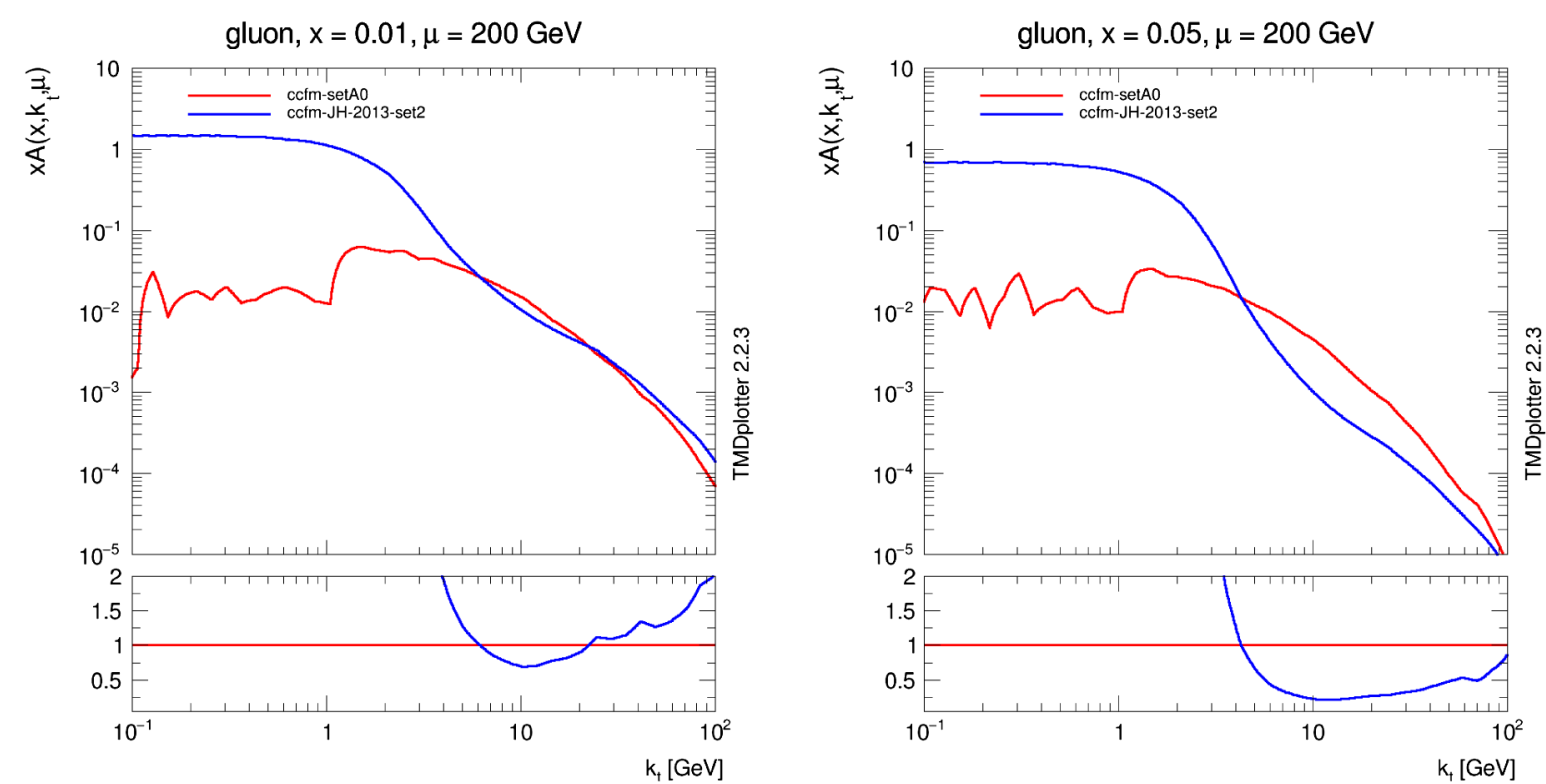

FIG. 1. A0 (red) and JH'2013 set 2 (blue) TMD gluon distributions in a proton as functions of the transverse momentum $k_{T}$ at different $x$ values and typical scale $\mu=200 \mathrm{GeV}$. The figure is made with TMDPlotter [62].

tool for our consideration because it smoothly interpolates between the small- $x$ BFKL gluon dynamics and conventional DGLAP one. Similarly to [52], to reconsruct one or a few leading hadronic jets from the initial-state gluon emissions, we have used the anti- $k_{T}$ algorithm with radia $R_{\text {jet }}$ as implemented in the FastJet tool [58].

Concerning the CCFM-evolved gluon densities in a proton, in the present note, we tested two different sets, ${ }^{3}$ namely, JH'2013 set 2 [60] and (more old) A0 set [61]. The input parameters of the latest gluon density, JH'2013 set 2, were derived from the best description of high-precision HERA data on proton structure functions $F_{2}\left(x, Q^{2}\right)$ and $F_{2}^{c}\left(x, Q^{2}\right)$ [60]. Both these TMD gluon distributions are shown in Fig. 1 as functions of gluon transverse momentum $k_{T}=\left|\mathbf{k}_{T}\right|$ at several values of $x$ and typical hard scale $\mu \sim m_{T} \sim 200 \mathrm{GeV}$. One can see some difference in the absolute normalization and shape between these gluon density functions. Below we consider the phenomenological consequences for associated Higgs and jets production at the LHC conditions.

Throughout this paper, all the calculations are based on the following parameter setting. We kept $n_{f}=4$ active (massless) quark flavors, set $\Lambda_{\mathrm{QCD}}=200(250) \mathrm{MeV}$, and used two-loop (one-loop) QCD coupling for JH'2013 set 2 (A0) gluon densities. As it is often done, the renormalization scale was taken to be $\mu_{R}^{2}=m_{H}^{2}$. The factorization scale was taken as $\mu_{F}^{2}=\hat{s}+\mathbf{Q}_{T}^{2}$ (where $\mathbf{Q}_{T}$ is the net transverse

\footnotetext{
${ }^{3} \mathrm{~A}$ comprehensive collection of TMD gluon densities can be found in the TMDlib package [59], which is a C++ library providing a framework and interface to different parametrizations.
}

momentum of the initial off-shell gluon pair) that is dictated mainly by the CCFM evolution algorithm (see [60,61] for more information). To estimate the theoretical uncertainties of our calculations, we use auxiliary gluon densities JH'2013 set 2+ and JH'2013 set 2- as well as $\mathrm{A} 0+$ and $\mathrm{A} 0-$ instead of the default gluon distribution functions. These two sets refer to the varied hard scales in the strong coupling constant $\alpha_{s}$ in the off-shell amplitude: "+" stands for $2 \mu_{R}$, while "-" refers to $\mu_{R} / 2$. Following [63], we set electroweak and Higgs bosons masses $m_{Z}=91.1876 \mathrm{GeV}, m_{W}=80.403 \mathrm{GeV}$, and $m_{H}=$ $125.1 \mathrm{GeV}$, their total decay widths $\Gamma_{Z}=2.4952 \mathrm{GeV}$, $\Gamma_{W}=2.085 \mathrm{GeV}$, and $\Gamma_{H}=4.3 \mathrm{MeV}$ and use $\sin ^{2} \theta_{W}=$ 0.23122 .

We start the discussion by presenting our results for associated Higgs boson and jet production in the diphoton decay channel. The latest measurements were done by the CMS [1] and ATLAS [5] Collaborations at the $\sqrt{s}=13 \mathrm{TeV}$. The applied experimental cuts are collected in Table I. An additional requirement (the isolation

TABLE I. Basic parameters, used for simulations in the $H \rightarrow \gamma \gamma$ decay channel.

\begin{tabular}{|c|c|c|}
\hline & ATLAS [5] & CMS [1] \\
\hline$p_{T}^{\gamma_{1}}, \mathrm{GeV}$ & $>0.35 m^{\gamma \gamma}$ & $>m^{\gamma \gamma} / 3$ \\
\hline$p_{T}^{\gamma_{2}}, \mathrm{GeV}$ & $>0.25 m^{\gamma \gamma}$ & $>m^{\gamma \gamma} / 4$ \\
\hline$\left|y^{\gamma}\right|$ & $<2.37$ & $<2.5$ \\
\hline$m^{\gamma \gamma}, \mathrm{GeV}$ & $105-160$ & $>90$ \\
\hline$R_{\text {jet }}$ & \multicolumn{2}{|c|}{0.4} \\
\hline$p_{T}^{\text {jet }}, \mathrm{GeV}$ & $>30$ & $>30$ \\
\hline$\left|y^{\text {jet }}\right|$ & $<4.4$ & $<4.7$ \\
\hline
\end{tabular}



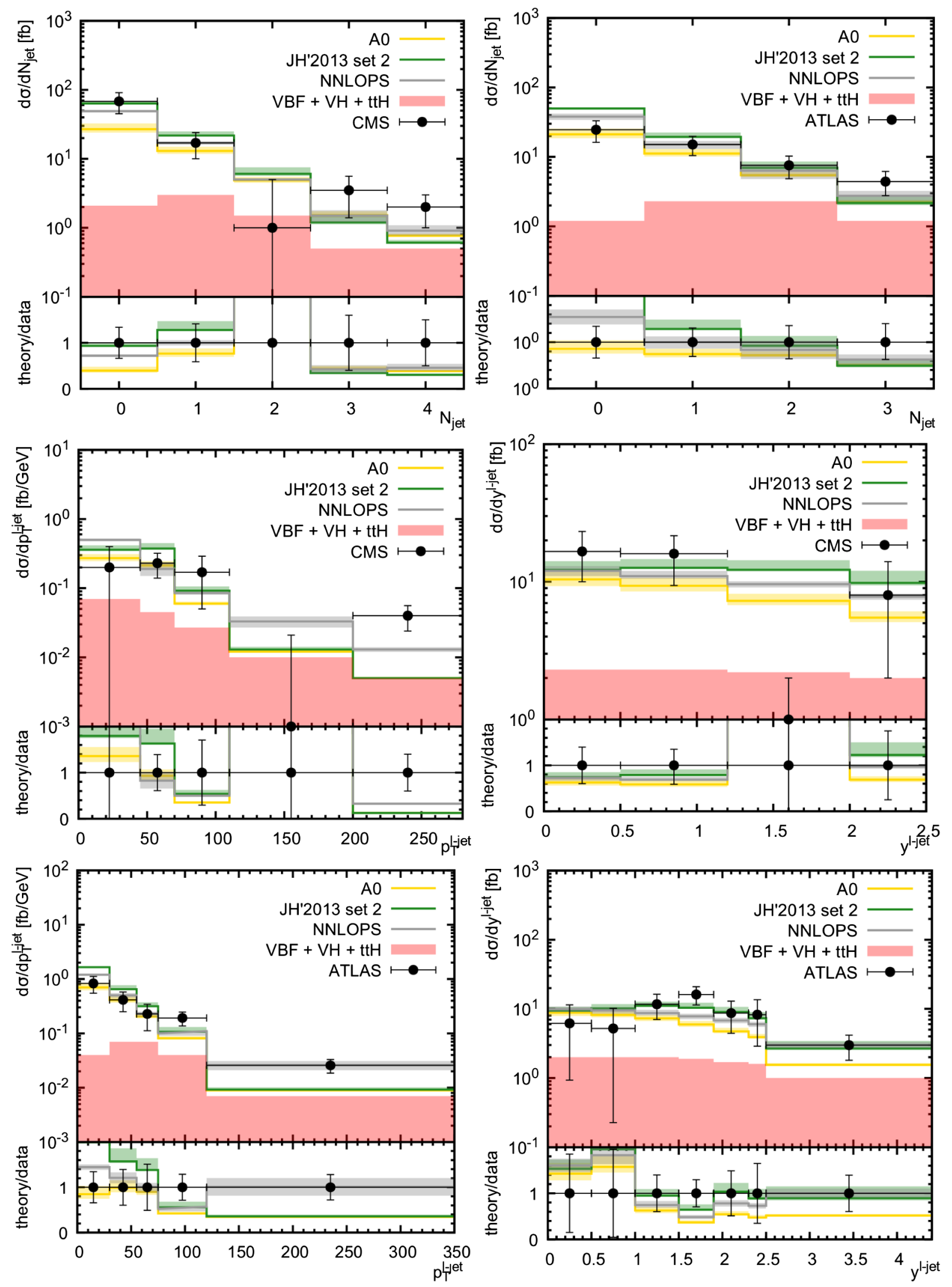

FIG. 2. The differential cross sections of associated Higgs boson and jet production (in the diphoton decay channel) at $\sqrt{s}=13$ TeV as functions of $N_{\text {jet }}$, leading jet transverse momentum and rapidity. The contributions from nongluon fusion subprocesses and NNLOPS predictions are taken from [1,5]. The experimental data are from CMS [1] and ATLAS [5]. 

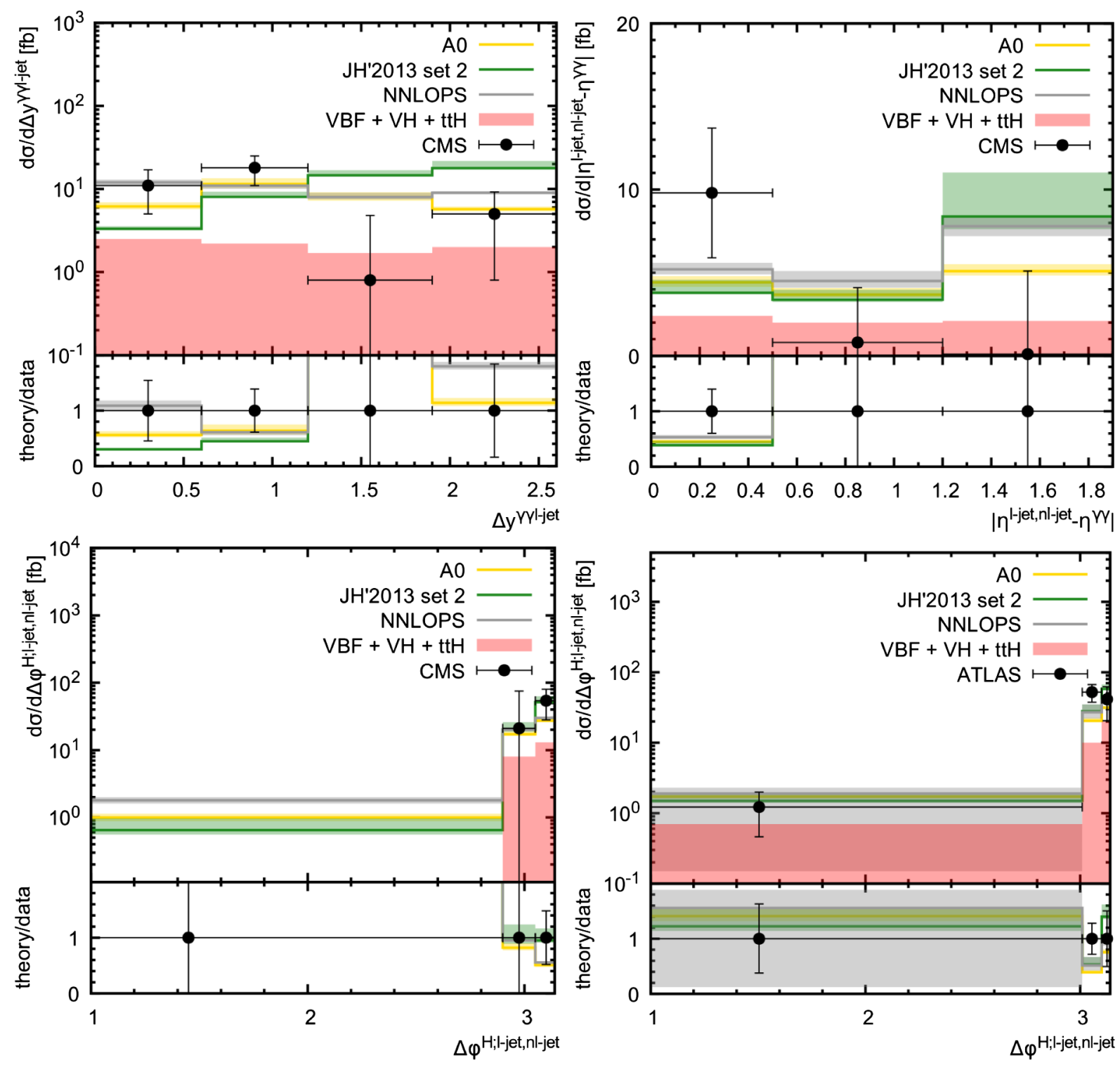

FIG. 3. The differential cross sections of associated Higgs boson and jet production (in the diphoton decay channel) at $\sqrt{s}=13$ TeV as functions of the rapidity difference between the diphoton system and leading jet $\Delta y^{\gamma \gamma j_{1}}$, Zeppenfeld variable $\left|\eta^{j_{1} j_{2}}-\eta^{\gamma \gamma}\right|$, and azimuthal angle difference between the diphoton and dijet systems $\Delta \phi^{H ; j_{1} j_{2}}$. The contributions from nongluon fusion subprocesses and NNLOPS predictions are taken from [1,5]. The experimental data are from CMS [1] and ATLAS [5].

criterion) is introduced for the photons in both experiments: the sum of transverse energy of particles around every photon within the radius $\Delta R=\sqrt{\Delta \eta^{2}+\Delta \phi^{2}}=0.3$ has to be smaller than $E_{\text {iso }}=10 \mathrm{GeV}$. The results of our calculations are presented on Figs. 2 and 3. Here we concentrated only on some of the kinematical variables among the quite large variety of those presented by the CMS and ATLAS Collaborations: number of jets $N_{\text {jet }}$, leading jet transverse momentum $p_{T}^{j_{1}}$ and rapidity $y^{j_{1}}$, rapidity difference between the diphoton system and leading jet, $\Delta y^{\gamma \gamma j_{1}}$, azimuthal angle difference between the diphoton and dijet system, $\Delta \phi^{H ; j_{1} j_{2}}$, and difference between the average pseudorapidity of these jets and pseudorapidity of the diphoton system $\left|\eta^{j_{1} j_{2}}-\eta^{\gamma \gamma}\right|$ (Zeppenfeld variable [64]). Note that we added to our results the contributions from weak boson fusion subprocesses $\left(W^{+} W^{-} \rightarrow H\right.$ and $Z Z \rightarrow H$ ), associated $H Z$ or $H W^{ \pm}$production, and associated $t \bar{t} H$ production. These contributions are essential at high transverse momenta and have been calculated in the conventional pQCD approach with the NLO accuracy (we took them from the CMS [1] and ATLAS [5] papers). Additionally, we show here the NNLO pQCD predictions, calculated with the NNLOPS program [17] and taken from $[1,5]$. As one can see, the measured cross sections can be reasonably well described by the $k_{T}$-factorization 
TABLE II. Basic parameters used for simulations in the $H \rightarrow Z Z^{*} \rightarrow 4 l$ decay channel. By default, experimental cuts for electrons are shown. Cuts for muons are placed in brackets, if differ.

\begin{tabular}{lcc}
\hline \hline & ATLAS [8] & CMS [4] \\
\hline$p_{T}^{l_{1}}, \mathrm{GeV}$ & $>22$ & $>25$ \\
$p_{T}^{l_{2}}, \mathrm{GeV}$ & $>15$ & $>13$ \\
$\left|\eta^{l}\right|$ & $<2.47$, excluding $1.37<\left|\eta^{l}\right|<1.52(<2.5)$ & $<2.5$ \\
$m^{l l}, \mathrm{GeV}$ & $10-55$ & 0.4 \\
$R_{\text {jet }}$ & $>25$, if $\left|\eta^{\text {jet }}\right|<2.4,>30$ otherwise & $>12$ \\
$p_{T}^{\text {jet }}, \mathrm{GeV}$ & $<4.5$ & $>30$ \\
$\left|\eta^{\text {jet }}\right|$ & $p_{T}^{\text {miss }}>20 \mathrm{GeV}$ & $p_{T}^{l l}>30 \mathrm{GeV}$ \\
Other cuts & $\Delta \phi^{l l}<1.8$ & $m_{T}^{l_{2}} \equiv \sqrt{2 p_{T}^{l_{2}} p_{T}^{\text {miss }}\left[1-\cos \Delta \phi\left(\mathbf{p}_{T}^{l_{2}}, \mathbf{p}_{T}^{\text {miss }}\right)\right]}>30 \mathrm{GeV}$ \\
& $m_{T}^{H} \equiv \sqrt{2 p_{T}^{l l} p_{T}^{\text {miss }}\left[1-\cos \Delta \phi\left(\mathbf{p}_{T}^{l l}, \mathbf{p}_{T}^{\text {miss }}\right)\right]}>60 \mathrm{GeV}$ \\
\hline \hline
\end{tabular}

evaluations based on both considered TMD gluon densities within the theoretical and experimental uncertainties. More "old" A0 gluon density tends to slightly underestimate the JH'2013 set 2 predictions although giving relatively larger number of jets at larger transverse momenta (see discussion below). However, we find that the predictions based on these gluon densities behave differently for some observables, especially for correlation ones, such as $\Delta y^{\gamma \gamma j_{1}}$ and azimuthal angle difference $\Delta \phi^{H ; j_{1} j_{2}}$. Moreover, the difference between these predictions exceeds the theoretical uncertainties of our calculations (see Fig. 2). Unfortunately, the current level of experimental accuracy does not allow us to favor one or another TMD gluon density in a proton. More precise future measurements of such observables could be promising to distinguish between the latter. The NNLO pQCD predictions behave similarly to the A0 results (except for distribution in $\Delta y^{\gamma \gamma j_{1}}$ ), though having larger normalization. The difference between the NNLO pQCD and JH'2013 set 2 results is clearly seen for $\Delta y^{\gamma \gamma j_{1}}$ and, in some sense, for $\Delta \phi^{H ; j_{1} j_{2}}$ observable. Nevertheless, our calculations demonstrate the possibility of $k_{T}$-factorization approach supplemented with the CCFM gluon dynamics to describe reasonably the collider data on events containing hadronic jets in final state.

Next, we turn to the $H \rightarrow Z Z^{*} \rightarrow 4 l$ and $H \rightarrow W^{+} W^{-} \rightarrow$ $e^{ \pm} \mu^{\mp} \nu \bar{\nu}$ decay modes. In these channels, the available experimental data were obtained by the CMS [2-4] and ATLAS [6-8] Collaborations at $\sqrt{s}=8$ and $13 \mathrm{TeV}$. The applied experimental cuts are listed in Tables II and III, respectively. Our results for several interesting observables, namely, $N_{\text {jet }}$, leading jet transverse momentum $p_{T}^{j_{1}}$, rapidity difference between the Higgs boson and leading jet, $\Delta y^{H j_{1}}$, pseudorapidity and azimuthal angle difference

TABLE III. Basic parameters used for simulations in the $H \rightarrow W^{+} W^{-} \rightarrow e^{ \pm} \mu^{\mp} \nu \bar{\nu}$ decay channel. By default, experimental cuts for electrons are shown. Cuts for muons are placed in brackets, if differ.

\begin{tabular}{|c|c|c|c|c|}
\hline & $\mathrm{CMS} \sqrt{s}=8 \mathrm{TeV}$ [2] & ATLAS $\sqrt{s}=8 \mathrm{TeV}[6]$ & $\mathrm{CMS} \sqrt{s}=13 \mathrm{TeV}[3]$ & ATLAS $\sqrt{s}=13 \mathrm{TeV}$ [7] \\
\hline Ordered $p_{T}^{l}, \mathrm{GeV}$ & $>20,10,7(5), 7(5)$ & $>20,15,10,7(6)$ & $>20,10,7(5), 7(5)$ & $>20,15,10,5$ \\
\hline$\left|\eta^{l}\right|$ & $<2.5(2.4)$ & $<2.7(2.47)$ & $<2.5(2.4)$ & $<2.7$ \\
\hline$m^{Z}$ & $40-120$ & $50-106$ & $40-120$ & $50-106$ \\
\hline$m^{Z^{*}}, \mathrm{GeV}$ & $12-120$ & $12-115$ & $12-120$ & $12-115$ \\
\hline$m^{4 l}, \mathrm{GeV}$ & $105-140$ & $118-129$ & $105-140$ & $105-160$ \\
\hline$\Delta R^{\text {same-sign leptons }}$ & $>0.02$ & $>0.1$ & $>0.02$ & $>0.1$ \\
\hline$\Delta R^{\text {opposite-sign leptons }}$ & $>0.02$ & $>0.2$ & $>0.02$ & $>0.1$ \\
\hline \multirow[t]{2}{*}{ Lepton isolation } & $\Delta R=0.4$ & & $\Delta R=0.3$ & \\
\hline & $E_{\text {iso }}=0$ & & $E_{\text {iso }}=0.35 p_{T}^{l}$ & \\
\hline$R_{\text {jet }}$ & 0.5 & 0.4 & 0.4 & 0.4 \\
\hline$p_{T}^{\text {jet }}, \mathrm{GeV}$ & $>30$ & $>30$ & $>30$ & $>30$ \\
\hline$\eta^{\mathrm{jet}}$ & $<4.7$ & $<4.4$ & $<2.5$ & $<4.4$ \\
\hline
\end{tabular}



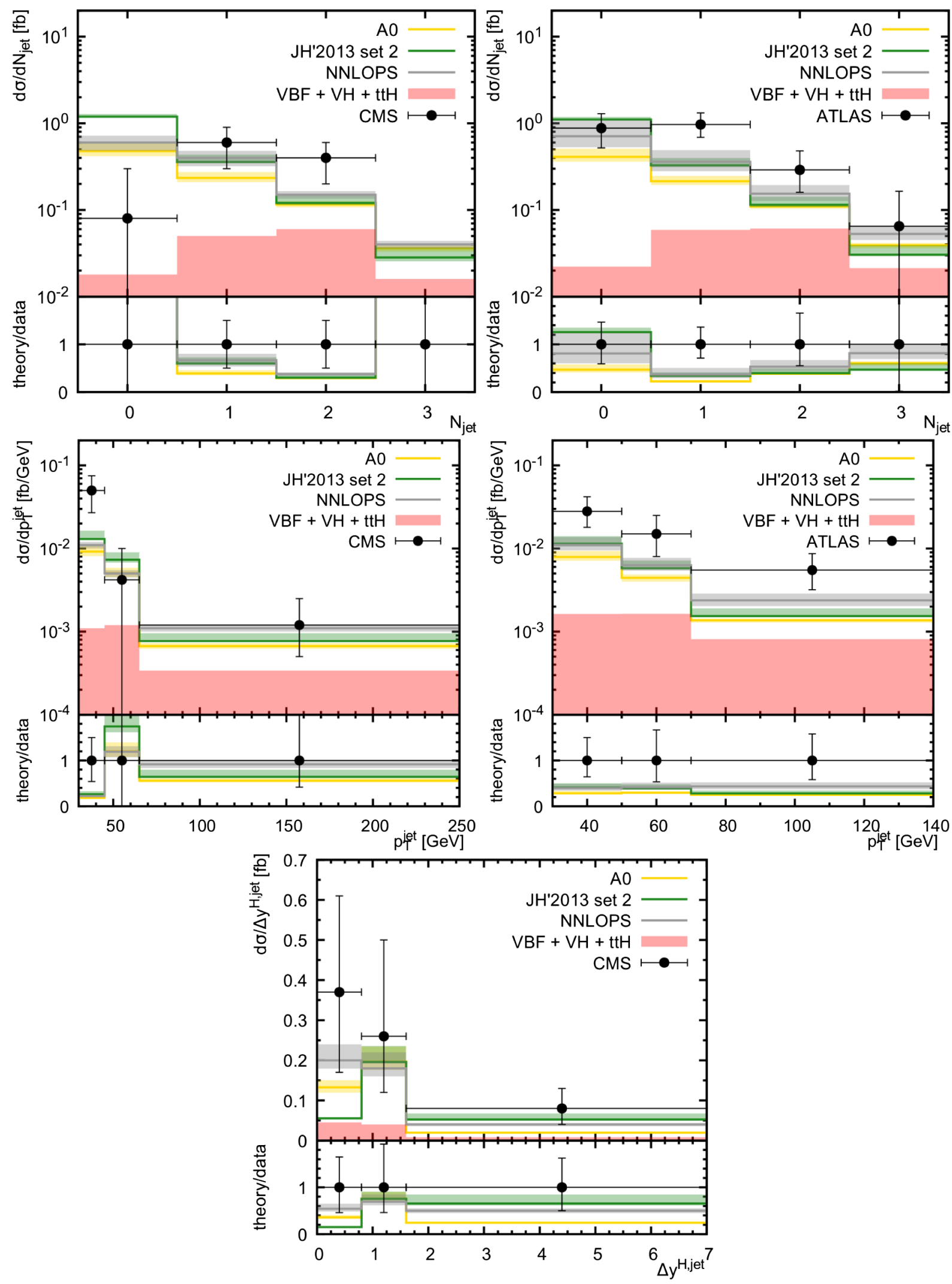

FIG. 4. The differential cross sections of associated Higgs boson and jet production (in the $H \rightarrow Z Z^{*}$ decay channel) at $\sqrt{s}=8 \mathrm{TeV}$ as functions of $N_{\text {jet }}$, leading jet transverse momentum, and rapidity difference between the Higgs boson and leading jet $\Delta y^{H j_{1}}$. The contributions from nongluon fusion subprocesses and NNLOPS predictions are taken from [2,6]. The experimental data are from CMS [2] and ATLAS [6]. 

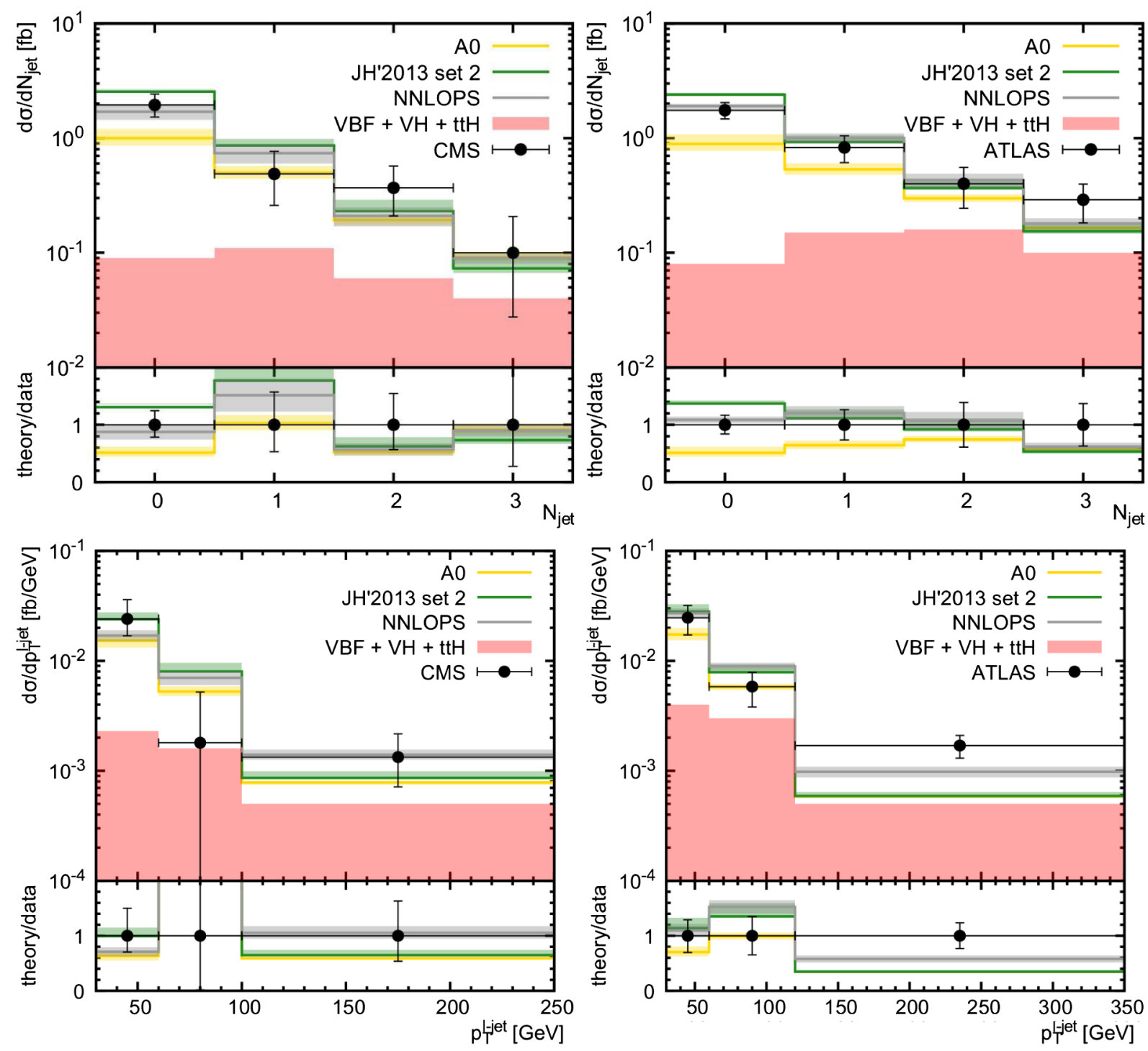

FIG. 5. The differential cross sections of associated Higgs boson and jet production (in the $H \rightarrow Z Z^{*}$ decay channel) at $\sqrt{s}=13$ TeV as functions of $N_{\text {jet }}$ and leading jet transverse momentum. The contributions from nongluon fusion subprocesses and NNLOPS predictions are taken from [3,7]. The experimental data are from CMS [3] and ATLAS [7].

between the leading and subleading jets, $\Delta \eta^{j_{1} j_{2}}$ and $\Delta \phi^{j_{1} j_{2}}$, invariant masses of Higgs-leading jet system $m^{H j_{1}}$ and Higgs-dijet system $m^{H j_{1} j_{2}}$, are shown in Figs. 4-7. As in the case of the diphoton decay mode, the contributions from the weak boson fusion subprocess, associated $H Z, H W^{ \pm}$, and $t \bar{t} H$ production calculated in the NLO pQCD approximation and taken from [2-4,6-8] were added to the off-shell gluon-gluon fusion. We find again that the latest JH'2013 set 2 gluon distribution reasonably well describes the LHC data within the estimated scale uncertainties, whereas A0 gluon tends to lack normalization. The measured data $[2-4,6-8]$ point to the following distinctive observables, which reveal the difference between JH'2013 set 2 and A0 gluon densities: Higgs-jet rapidity difference $\Delta y^{H j_{1}}$, difference in pseudorapidity between the leading and subleading jets $\Delta \eta^{j_{1} j_{2}}$ and invariant masses $m^{H j_{1}}$ and $m^{H j_{1} j_{2}}$. The latter demonstrate much larger cross section at relatively low invariant masses for A0 predictions, which is, in fact, in better agreement with the ATLAS data (see Fig. 5). Thus, in more precise forthcoming experiments, the highlighted observables, in addition to the variables for inclusive Higgs production pointed earlier [41,46,49-51], could be promising to distinguish between the different TMD gluon densities or to better constrain their parameters. Like as for $H \rightarrow \gamma \gamma$ decay channel, we plot also the collinear NNLO pQCD results, taken from [2-4,6-8]. It can be seen again that the A0 distributions generally follow the collinear results in shape, whereas the JH'2013 set 2 results somewhat differ from them.

Finally, we also make a comparison with the results of fixed-order NNLO QCD in collinear factorization. In 

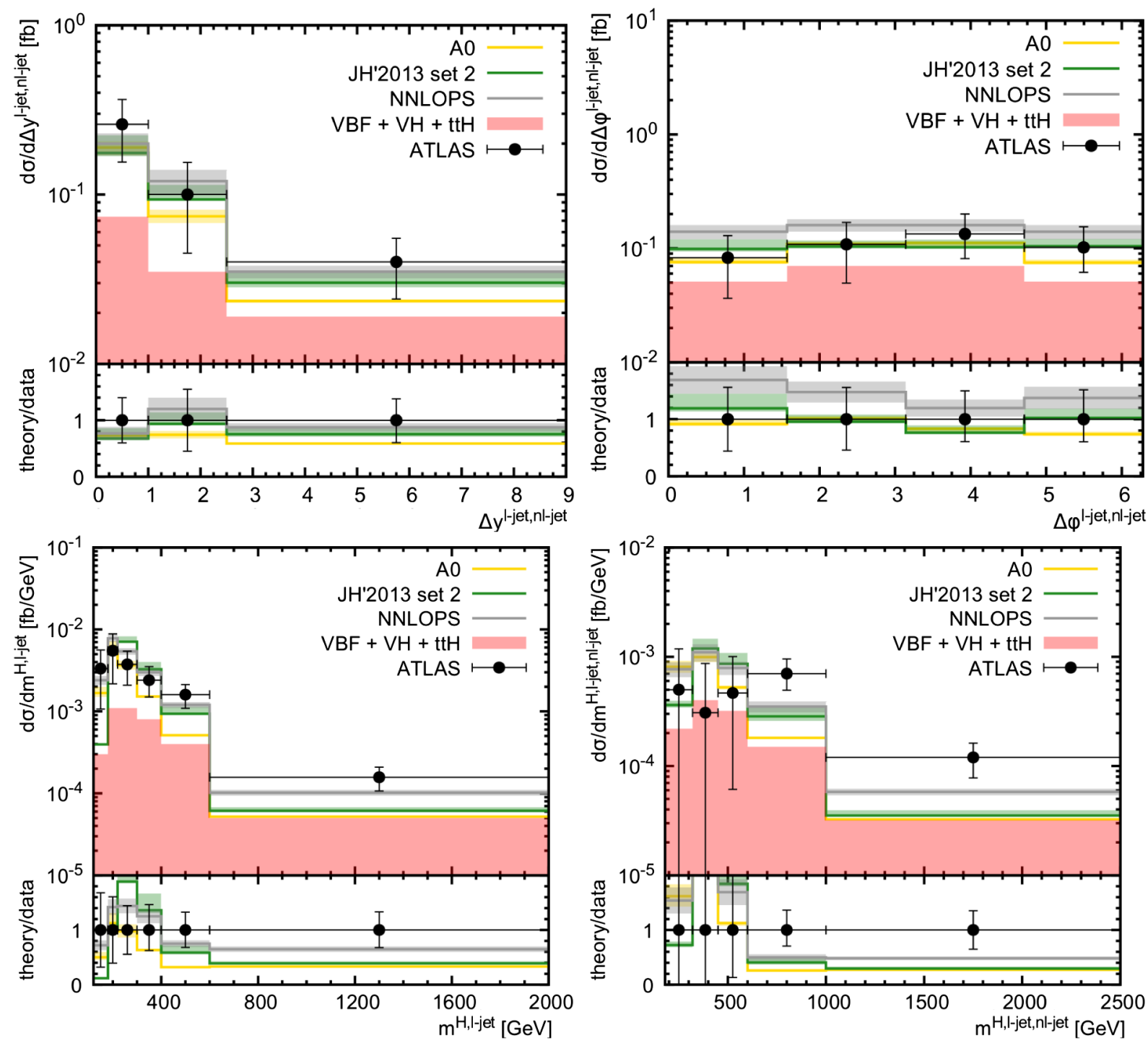

FIG. 6. The differential cross sections of associated Higgs boson and jet production (in the $H \rightarrow Z Z^{*}$ decay channel) at $\sqrt{s}=13$ TeV as functions of the rapidity and azimuthal angle difference between the leading and subleading jets, invariant masses of Higgs-leading jet system and Higgs-dijet system. The contributions from nongluon fusion subprocesses and NNLOPS predictions are taken from [3,7]. The experimental data are from CMS [3] and ATLAS [7].

Fig. 8, we show differential cross sections obtained in our approach with JH'2013 set 2 and compare them with the results taken from [20]. The calculations are made at $\sqrt{s}=$ $8 \mathrm{TeV}$ for $H \rightarrow \gamma \gamma$ decay channel, and we adopt all the kinematical restrictions from [20]. Note that here we take into account only the gluon-fusion contribution. One can see that in general jet multiplicity and rapidity distributions obtained in different approaches agree with each other, although uncertainties in NNLO case are typically significantly smaller (as one should have expected for higherorder calculations). The distributions for $p_{T}^{\text {jet }}$ are however very different: the $k_{T}$-factorization cross section shows much steeper behavior. This is in agreement with the results shown in Figs. 2, 4, and 5. Therefore, one can expect, that a consistent treatment of higher-order corrections in the $k_{T^{-}}$ factorization would significantly improve description of the data. ${ }^{4}$

Let us a little further discuss the obtained results. The uncertainty connected with the choice of the TMD gluon density appears to be rather significant for some observables. This is similar to the situation that was some decades ago in the standard collinear (DGLAP-based) approach, when different parton distributions sets could vary largely and lead to different predictions. This reflects, in some sense, the current situation in the $k_{T}$-factorization, where, in our opinion, further efforts in the determination of TMD

\footnotetext{
${ }^{4}$ Recently, large progress has been made in calculating NLO corrections for Higgs boson production within $k_{T^{-}}$ factorization $[65,66]$.
} 

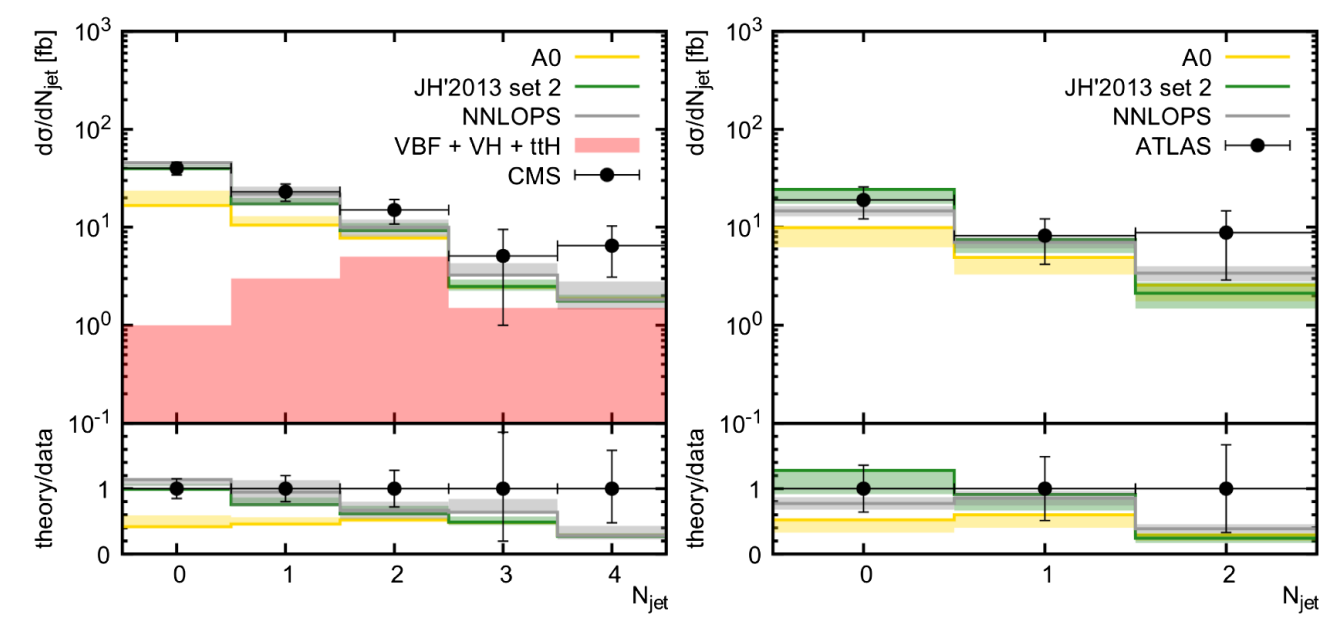

FIG. 7. The differential cross sections of associated Higgs boson and jet production (in the $H \rightarrow W^{+} W^{-}$decay channel) at $\sqrt{s}=8$ (right panel) and $13 \mathrm{TeV}$ (left panel) as functions of $N_{\text {jet }}$. The experimental data are from CMS [4] and ATLAS [8].
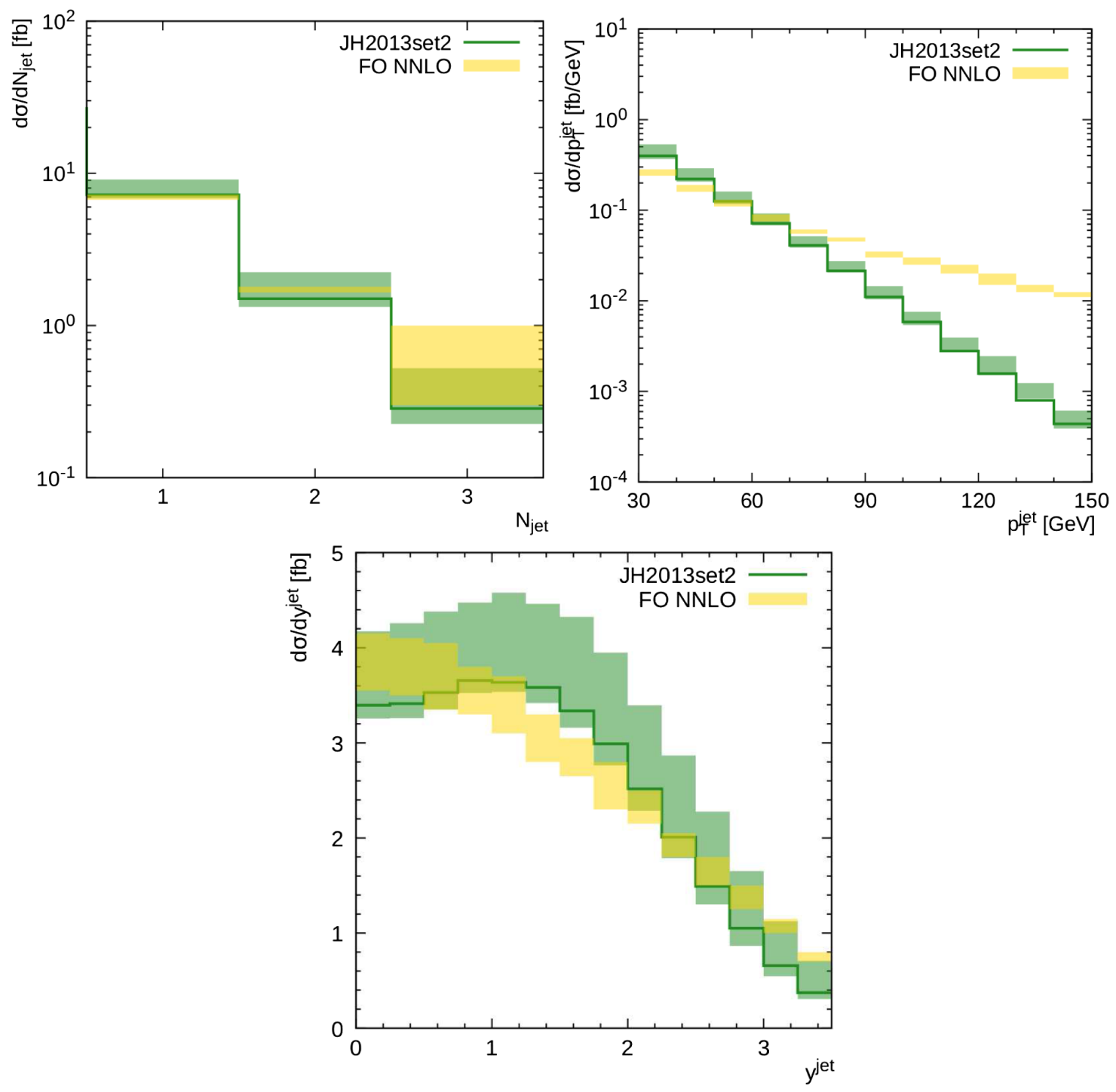

FIG. 8. The differential cross sections of associated Higgs boson and jet production (in the $H \rightarrow \gamma \gamma$ decay channel) at $\sqrt{s}=8$ as functions of $N_{\text {jet }}, p_{T}^{1 \text {-jet }}, y^{\text {jet }}$. The fixed-order NNLO QCD results are taken from [20]. 
parton distributions are needed. So far, neither of the used CCFM TMDs gives good agreement with the data in all kinematical range for all observables. The reason of the clear difference between the predictions of the JH'2013 set 2 and $\mathrm{A} 0$ gluons comes from their different $k_{T}$-behavior. In fact, the JH'2013 set 2 gluon distribution yields larger normalization, but at larger $k_{T}$ the A0 density tends to be dominating one (see Fig. 1). This means that the Higgs production cross section in the A0 case is dominated by events, in which the incoming gluons have quite large transverse momenta. These transverse momenta are compensated by the transverse momenta of the cascade gluons, giving rise eventually to jets. This results in the fact that in comparison with the JH'2013 set 2 case, one sees harder jets and larger number of hard jets. As long as one has larger number of jets, one expects them to be more uniformly distributed in the transverse plane, thus smearing $\Delta \phi$ distributions. All these features are seen in Figs. 2-7. So, the associated Higgs + jets production at the LHC can be used as an additional good probe of the TMD gluon density in a proton.

To conclude, we calculated for the first time the cross sections of associated Higgs and jet(s) production at the LHC conditions using the $k_{T}$-factorization approach. Our consideration covers different Higgs decay channels and is mainly based on the off-shell production amplitudes for the gluon-gluon fusion subprocess (implemented into the Monte Carlo event generator PEGASUS) and CCFM-evolved TMD gluon densities in a proton. To reconstruct correctly the kinematics of the final-state hadronic jets, the TMD parton shower generator CASCADE has been applied. Our predictions obtained with the recent JH'2013 set 2 gluon density agree well with the experimental data taken by the CMS and ATLAS Collaborations at $\sqrt{s}=8$ and $13 \mathrm{TeV}$. We have found observables, which are sensitive to the TMD gluon densities in a proton. As it was expected, these are the ones related with the properties of the produced jets, for example, the rapidity difference between the Higgs boson and leading jet. Unfortunately, the current level of experimental accuracy does not allow to distinguish between the latter. However, more precise future experimental studies of the pointed observables could be promising and could allow one to constrain the TMD gluons. Our study demonstrates the possibility of $k_{T^{-}}$ factorization approach supplemented with the CCFM gluon dynamics to describe the events with large number of jets in final state. It significantly extends the previous consideration $[41,46,49]$ of inclusive Higgs production at the LHC.

We thank S. P. Baranov and H. Jung for their interest, very useful discussions on the topic and important remarks. We also thank X. Chen for providing us with results of their calculations. We are grateful to the DESY Directorate for the support in the framework of Cooperation Agreement between MSU and DESY on phenomenology of the LHC processes and TMD parton densities. The work of M. A. M. was also supported by the grant of the Foundation for the Advancement of Theoretical Physics and Mathematics "BASIS" 20-1-3-11-1.
[1] CMS Collaboration, J. High Energy Phys. 01 (2019) 183.

[2] CMS Collaboration, J. High Energy Phys. 04 (2016) 005.

[3] CMS Collaboration, J. High Energy Phys. 11 (2017) 047.

[4] CMS Collaboration, J. High Energy Phys. 03 (2021) 003.

[5] ATLAS Collaboration, Phys. Rev. D 98, 052005 (2018).

[6] ATLAS Collaboration, Phys. Lett. B 738, 234 (2014).

[7] ATLAS Collaboration, Eur. Phys. J. C 80, 942 (2020).

[8] ATLAS Collaboration, J. High Energy Phys. 08 (2016) 104.

[9] LHC Higgs Cross Section Working Group, arXiv:1610. 07922.

[10] M. Spira, A. Djouadi, D. Graudenz, and P. M. Zerwas, Nucl. Phys. B453, 17 (1995).

[11] A. Djouadi, M. Spira, and P. M. Zerwas, Phys. Lett. B 264, 440 (1991).

[12] S. Dawson, Nucl. Phys. B359, 283 (1991).

[13] R. V. Harlander and W. B. Kilgore, Phys. Rev. Lett. 88, 201801 (2002).

[14] C. Anastasiou and K. Melnikov, Nucl. Phys. B646, 220 (2002).

[15] V. Ravindran, J. Smith, and W. L. van Neerven, Nucl. Phys. B665, 325 (2003).
[16] D. de Florian, G. Ferrera, M. Grazzini, and D. Tommasini, J. High Energy Phys. 06 (2012) 132.

[17] K. Hamilton, P. Nason, E. Re, and G. Zanderighi, J. High Energy Phys. 10 (2013) 222.

[18] R. Boughezal, F. Caola, K. Melnikov, F. Petriello, and M. Schulze, J. High Energy Phys. 06 (2013) 072.

[19] R. Boughezal, F. Caola, K. Melnikov, F. Petriello, and M. Schulze, Phys. Rev. Lett. 115, 082003 (2015).

[20] F. Caola, K. Melnikov, and M. Schulze, Phys. Rev. D 92, 074032 (2015).

[21] X. Chen, T. Gehrmann. E. W. N. Glover, and M. Jaquier, Phys. Lett. B 740, 147 (2015).

[22] X. Chen, J. Cruz-Martinez, T. Gehrmann, E. W. N. Glover, and M. Jaquier, J. High Energy Phys. 10 (2016) 066.

[23] M. Grazzini, S. Kallweit, and M. Wiesemann, Eur. Phys. J. C 78, 537 (2018).

[24] X. Chen, T. Gehrmann, E. W. N. Glover, and A. Huss, J. High Energy Phys. 07 (2019) 052.

[25] C. Anastasiou, C. Duhr, F. Dulat, E. Furlan, T. Gehrmann, F. Herzog, A. Lazopoulos, and B. Mistlberger, J. High Energy Phys. 05 (2016) 058. 
[26] X. Chen, T. Gehrmann. E. W. N. Glover, A. Huss, B. Mistlberger, and A. Pelloni, arXiv:2102.07607.

[27] U. Aglietti, R. Bonciani, G. Degrassi, and A. Vicini, Phys. Lett. B 595, 432 (2004).

[28] S. Actis, G. Passarino, C. Sturm, and S. Uccirati, Phys. Lett. B 670, 12 (2008).

[29] D. de Florian and M. Grazzini, Phys. Lett. B 718, 117 (2012).

[30] C. Anastasiou, S. Buehler, F. Herzog, and A. Lazopoulos, J. High Energy Phys. 04 (2012) 004.

[31] J. Baglio and A. Djouadi, J. High Energy Phys. 03 (2011) 055 .

[32] S. Catani, D. de Florian, M. Grazzini, and P. Nason, J. High Energy Phys. 07 (2003) 028.

[33] D. de Florian, G. Ferrera, M. Grazzini, and D. Tommasini, J. High Energy Phys. 11 (2011) 064.

[34] S. Catani, M. Ciafaloni, and F. Hautmann, Nucl. Phys. B366, 135 (1991); J. C. Collins and R. K. Ellis, Nucl. Phys. B360, 3 (1991).

[35] L. V. Gribov, E. M. Levin, and M. G. Ryskin, Phys. Rep. 100, 1 (1983); E. M. Levin, M. G. Ryskin, Yu. M. Shabelsky, and A. G. Shuvaev, Sov. J. Nucl. Phys. 53, 657 (1991).

[36] E. A. Kuraev, L. N. Lipatov, and V. S. Fadin, Sov. Phys. JETP 44, 443 (1976); 45, 199 (1977); I. I. Balitsky and L. N. Lipatov, Sov. J. Nucl. Phys. 28, 822 (1978).

[37] M. Ciafaloni, Nucl. Phys. B296, 49 (1988); S. Catani, F. Fiorani, and G. Marchesini, Phys. Lett. B 234, 339 (1990); Nucl. Phys. B336, 18 (1990); Nucl. Phys. B445, 49 (1995).

[38] R. Angeles-Martinez et al., Acta Phys. Pol. B 46, 2501 (2015).

[39] Small-x Collaboration, Eur. Phys. J. C 25, 77 (2002); 35, 67 (2004); 48, 53 (2006).

[40] A. Gawron and J. Kwiecinski, Phys. Rev. D 70, 014003 (2004).

[41] A. V. Lipatov and N. P. Zotov, Eur. Phys. J. C 44, 559 (2005).

[42] R. S. Pasechnik, O. V. Teryaev, and A. Szczurek, Eur. Phys. J. C 47, 429 (2006).

[43] G. Watt, A. D. Martin, and M. G. Ryskin, Phys. Rev. D 70, 014012 (2004).
[44] F. Hautmann, Phys. Lett. B 535, 159 (2002).

[45] H. Jung, Mod. Phys. Lett. A 19, 1 (2004).

[46] A. V. Lipatov, M. A. Malyshev, and N. P. Zotov, Phys. Lett. B 735, 79 (2014).

[47] R. Islam, M. Kumar, and V. S. Rawoot, Eur. Phys. J. C 79, 181 (2019).

[48] A. Szczurek, M. Luszczak, and R. Maciula, Phys. Rev. D 90, 094023 (2014).

[49] N. A. Abdulov, A. V. Lipatov, and M. A. Malyshev, Phys. Rev. D 97, 054017 (2018)

[50] N. A. Abdulov, H. Jung, A. V. Lipatov, G. I. Lykasov, and M. A. Malyshev, Phys. Rev. D 98, 054010 (2018).

[51] A. V. Kotikov, A. V. Lipatov, B. G. Shaikhatdenov, and P. Zhang, J. High Energy Phys. 02 (2020) 028.

[52] S. Dooling, F. Hautmann, and H. Jung, Phys. Lett. B 736, 293 (2014).

[53] H. Jung, S. P. Baranov, M. Deak, A. Grebenyuk, F. Hautmann, M. Hentschinski, A. Knutsson, M. Kramer, K. Kutak, A. V. Lipatov, and N. P. Zotov, Eur. Phys. J. C 70, 1237 (2010).

[54] J. R. Ellis, M. K. Gaillard, and D. V. Nanopoulos, Nucl. Phys. B106, 292 (1976).

[55] M. A. Shifman, A. I. Vainstein, M. B. Voloshin, and V. I. Zakharov, Sov. J. Nucl. Phys. 30, 711 (1979).

[56] A. V. Lipatov, M. A. Malyshev, and S. P. Baranov, Eur. Phys. J. C 80, 330 (2020).

[57] J. Alwall et al., Comput. Phys. Commun. 176, 300 (2007).

[58] M. Cacciari, G. P. Salam, and G. Soyez, Eur. Phys. J. C 72, 1896 (2012).

[59] F. Hautmann, H. Jung, M. Krämer, P. J. Mulders, E. R. Nocera, T. C. Rogers, and A. Signori, Eur. Phys. J. C 74, 3220 (2014).

[60] F. Hautmann and H. Jung, Nucl. Phys. B883, 1 (2014).

[61] H. Jung, arXiv:hep-ph/0411287.

[62] http://tmdplotter.desy.de.

[63] PDG Collaboration, Phys. Rev. D 98, 030001 (2018).

[64] D. L. Rainwater, R. Szalapski, and D. Zeppenfeld, Phys. Rev. D 54, 6680 (1996).

[65] M. A. Nefedov, J. High Energy Phys. 08 (2020) 055.

[66] M. Hentschinski, K. Kutak, and A. van Hameren, Eur. Phys. J. C 81, 112 (2021); 81, 262(E) (2021). 